\title{
Guest Editorial: Remote Sensing of the Wenchuan Earthquake
}

\author{
Huadong Guo \\ Center for Earth Observation and Digital Earth, Chinese Academy of Sciences, Laboratory of \\ Digital Earth Science, Beijing, 100190 China \\ hdguo@ceode.ac.cn
}

On May 12, 2008, Wenchuan County in the Sichuan Province of China experienced a severe earthquake of magnitude 8.0 on the Richter scale, causing heavy loss of life and property. After the tremor first hit, roads were blocked, communications went down, many secondary disasters occurred, and weather conditions were very bad. The lack of information was a serious problem for disaster relief and decision making. Urgent monitoring and assessment of the disaster situation were extremely vital, and there was an imperative need for scientific support for the search and rescue operations.

For this urgent time, a Project Team of Remote Sensing Monitoring and Assessment for the Wenchuan Earthquake was set up, consisting of experts from seven institutes of the Chinese Academy of Sciences (CAS). A project was immediately initiated to acquire data from earth observation satellites, and two high-altitude aircrafts were sent to acquire highresolution optical and radar data. On-the-spot field investigations were also promptly carried out. In this way, a unified, all-weather, day and night monitoring grid was established with spaceborne, airborne, and in-situ observing capacities. The disaster data analysis group worked continuously around the clock, collecting, transferring, processing, and interpreting remote sensing data and images, and promptly completing a series of analysis reports that were immediately submitted to the Headquarters for Earthquake Resistance and Disaster Relief of governments at the national, ministerial, and provincial levels. The delivered reports provided convincing evidence for the decision makers.

The articles in this special section were written by the main members of the CAS Project Team of Remote Sensing Monitoring and Assessment for the Wenchuan Earthquake, based on monitoring and assessing the disaster situation using the following earth observation data: ADS40, ALOS PALSAR, SPOT, Landsat, TerraSAR, RADARSAT-1, RADARSAT-2, as well as some other remote sensing imagery. The main research issues presented in this special section can be summarized as follows:

1. Methodology for information extraction. Some methods are proposed for detecting and extracting earthquake disaster information such as collapsed buildings, landslides, dammed palaeolakes, land surface changes, and so on based on air- and spaceborne synthetic aperture radar and optical images.

2. Evaluation for earthquake disaster. After the earthquake, many secondary disasters happened such as landslides, dammed palaeolakes, blocked transportation networks, collapsed houses and buildings, etc. We monitored, analyzed, and evaluated the spatial distribution and extent of those disasters in real time based on massive remote sensing data and advanced analytical models and methods.

3. Simulation. At the same time, a simulation system of the Wenchuan earthquake zone was developed to analyze the disaster situation and manage the massive amount of remote sensing data and various metadata. 